\title{
CONTROLLING THE SYNCHRONIZATION OF FORCED SELF-SUSTAINED OSCILLATIONS *
}

\author{
T. Pyragienė and K. Pyragas \\ Semiconductor Physics Institute, A. Goštauto 11, LT-01108 Vilnius, Lithuania \\ E-mail: pyragiene@pfi.lt
}

Received 30 June 2005

\begin{abstract}
The delayed feedback control method is applied to control a quasi-periodic motion. We consider a weakly nonlinear van der Pol oscillator subjected to a periodic force. Making use of the fact that the system is close to a supercritical Hopf bifurcation we are able to treat it analytically. Our analysis shows that the domain of synchronization of a forced self-sustained oscillator can be essentially extended by delayed feedback. The main results and the approach are of general importance since they are relevant to any forced self-sustained oscillator close to the supercritical Hopf bifurcation.
\end{abstract}

Keywords: delayed feedback control, Hopf bifurcation, synchronization

PACS: $05.45 . \mathrm{Gg}, 02.30 . \mathrm{Yy}, 02.30 . \mathrm{Ks}$

\section{Introduction}

The delayed feedback control (DFC) method [1] is superior for controlling the oscillations in applied nonlinear science [2]. The method allows a noninvasive stabilization of unstable periodic orbits (UPOs) of dynamical systems. The DFC makes use of a control signal obtained from the difference between the current state of the system and the state of the system delayed by one period of the unstable orbit.

Most investigations in the theory of delayed feedback control are devoted to the stabilization of unstable periodic orbits embedded in chaotic attractors of low-dimensional (usually three-dimensional) systems [4]. The leading Floquet multipliers of such orbits are real-valued and lay outside the unit circle in the complex plane (Fig. 1(a,b)). However, there exists a large class of unstable periodic orbits with the complex conjugate pair of leading Floquet multipliers (Fig. 1(c)). Such orbits arise from a Neimark-Sacker (discrete Hopf) bifurcation and cannot appear in lowdimensional chaotic attractors. Presumably for this reason they have not been considered in delayed feedback control theory so far. However, such orbits may appear in low-dimensional non-chaotic systems. In this paper we consider the control of such orbits.

\footnotetext{
* The report presented at the 36th Lithuanian National Physics Conference, 16-18 June 2005, Vilnius, Lithuania.
}
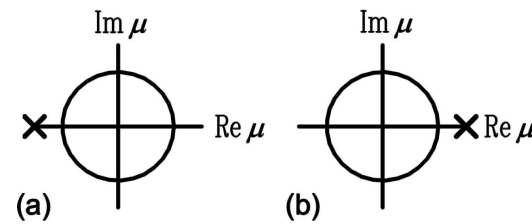

(b)

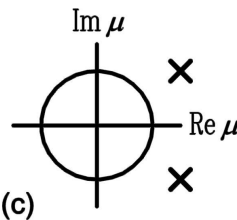

Fig. 1. Leading Floquet multipliers of unstable periodic orbits arising from different bifurcations: (a) period doubling, (b) tangent, and (c) Neimark-Sacker (discrete Hopf) bifurcations. The unit circle defines the region of stability.

We investigate the problem of controlling synchronization in a forced self-sustained oscillator. Very often in practical application the need arises to control the properties of oscillations. Usually control assumes an enhancement in regularity of motion. Due to drift of parameters the desired synchronization may be lost and a kind of beat phenomenon may occur. We imagine that the external force driving the oscillator is inaccessible in experiment, but we can measure an output of the oscillator and can control its state through some accessible input. Then applying the delayed feedback control we can return the system to the synchronized state. In this paper, we demonstrate these ideas for the van der Pol oscillator.

The rest of the paper is organized as follows. In Section 2 we derive averaged equations for the controlled van der Pol oscillator. In Section 3 we analyse periodic orbits of the uncontrolled system. In Section 4, we investigate a linear stability of the unstable periodic 
orbits controlled by delayed feedback. In Section 5, we demonstrate the results of numerical simulations of the original delay-differential equations. The paper is finished with conclusions presented in Section 6.

\section{Model and averaged equation}

Consider a weakly nonlinear van der Pol oscillator under the action of an external periodic force and a delayed feedback perturbation:

$$
\ddot{x}+\omega_{0}^{2} x+\varepsilon\left(x^{2}-1\right) \dot{x}=a \sin (\omega t)+k\left(x-x_{T}\right) .
$$

The left-hand side represents the standard van der Pol equation. The parameter $\omega_{0}$ is the characteristic frequency of self-sustained oscillations, and $\varepsilon$ is responsible for the strength of nonlinearity of the oscillator. The first term in the right-hand side is an external periodic force ( $a$ is the amplitude and $\omega$ is the frequency), and the second term describes the delayed coupling due to control. The parameter $k$ is the feedback gain, $x_{T} \equiv x(t-T)$, and $T=2 \pi / \omega$ is the period of the external force. In the following we consider Eq. (1) as a weakly nonlinear system. Specifically, we suppose that $\varepsilon$ is a small parameter, $\varepsilon \ll \omega_{0}$. Moreover, we assume that the amplitude $a$, the frequency detuning $\omega-\omega_{0}$, as well as the control perturbation $k\left(x-x_{T}\right)$ are proportional to the small parameter $\varepsilon$.

For weakly nonlinear systems, there are many mathematically rigorous ways (e.g., method of averaging, multiscale expansion, and other asymptotic methods) to obtain approximate solutions. We will apply the method of averaging. First we rewrite Eq. (1) as a system

$$
\begin{aligned}
\dot{x}= & y, \\
\dot{y}= & -\omega_{0}^{2} x-\varepsilon\left(x^{2}-1\right) y+a \sin (\omega t) \\
& +k\left(x-x_{T}\right) .
\end{aligned}
$$

As Eq. (1) or system (2) is close to that of linear oscillator, we may expect that the solution has a nearly harmonic form. Since there is a forced system, we look for a solution with the characteristic frequency $\omega$,

$$
x=\frac{A(t) \mathrm{e}^{\mathrm{i} \omega t}+A^{*}(t) \mathrm{e}^{-\mathrm{i} \omega t}}{2} .
$$

Here $A(t)$ is a new variable, a slowly varying complex amplitude. Since it is complex, we need two relations to have one-to-one correspondence between $(x, y)$ and
$A$. It is convenient to introduce the following relation between $y$ and $A$ :

$$
y=\mathrm{i} \omega \frac{A(t) \mathrm{e}^{\mathrm{i} \omega t}-A^{*}(t) \mathrm{e}^{-\mathrm{i} \omega t}}{2} .
$$

Substituting Eqs. (3) and (4) in system (2) we obtain the equation for the complex amplitude, which after averaging over the period $T$ of fast oscillations takes the form

$$
\begin{aligned}
\dot{A}= & \frac{\omega^{2}-\omega_{0}^{2}}{2 \mathrm{i} \omega} A-\frac{\varepsilon}{2} A\left(\frac{|A|^{2}}{4}-1\right)-\frac{a}{2 \omega} \\
& +\frac{k}{2 \mathrm{i} \omega}\left(A-A_{T}\right) .
\end{aligned}
$$

By choosing an appropriate scale for the amplitude

$$
A=2 z
$$

and introducing new parameters

$$
\alpha=\frac{a}{2 \varepsilon \omega}, \nu=\frac{\omega^{2}-\omega_{0}^{2}}{\varepsilon \omega} \approx 2 \frac{\omega-\omega_{0}}{\varepsilon}, \kappa=\frac{k}{\varepsilon \omega},
$$

equation (5) can be simplified to

$$
\frac{2}{\varepsilon} \dot{z}=-\mathrm{i} \nu z-z\left(|z|^{2}-1\right)-\alpha-\mathrm{i} \kappa\left(z-z_{T}\right) .
$$

The parameters $\alpha, \nu$, and $\kappa$ are proportional to the amplitude of external force, the frequency detuning, and the delayed feedback gain respectively. The dynamical equation for the complex amplitude (8) is the main instrument in our analysis of the stationary solutions of the system (2).

\section{Periodic orbits of the system}

We now determine the steady state solutions of the uncontrolled system $(\kappa=0)$ and analyse their stability. We focus on unstable periodic orbits. In the following sections they will be the subject of the delayed feedback control.

We start the analysis with finding the stationary solutions. Setting $\dot{z}=0$ and $z=z_{0}$, we obtain

$$
-\mathrm{i} \nu z_{0}-z_{0}\left(\left|z_{0}\right|^{2}-1\right)-\alpha=0 .
$$

We introduce the notations

$$
s=\left|z_{0}\right|^{2}, \quad f_{\nu}(s)=s\left[(s-1)^{2}+\nu^{2}\right] .
$$

Then the values of $s$ can be found by solving the cubic equation

$$
f_{\nu}(s)=\alpha^{2}
$$




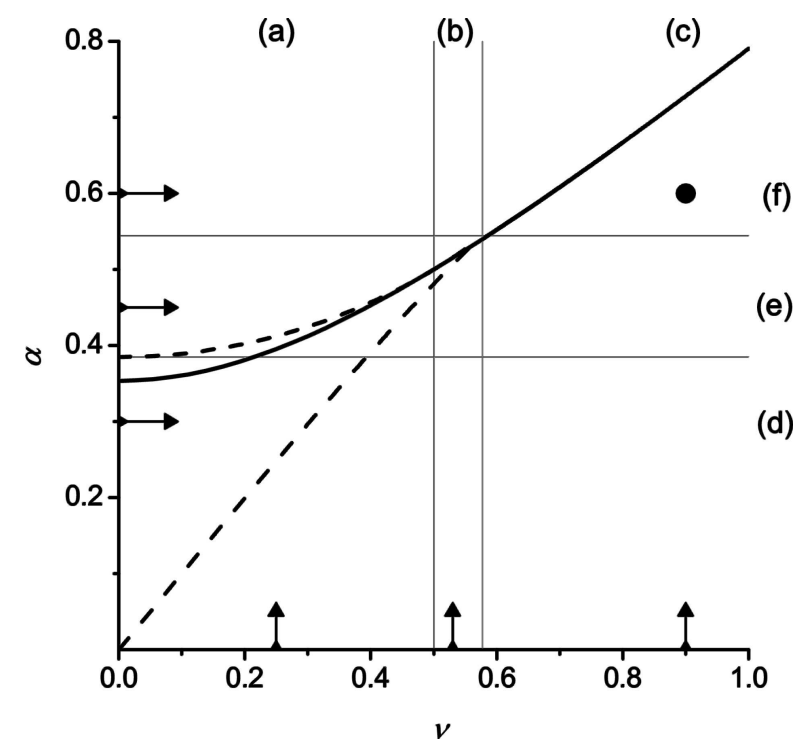

Fig. 2. The bifurcation diagram for the uncontrolled van der Pol oscillator. The broken lines are defined by Eq. (14b). The region between these lines correspond to three periodic orbits. Outside this region there is only one periodic orbit. The thick solid line is the hyperbola (17) defining the Hopf bifurcation. The vertical lines divide the diagram into three regions: (a) $\nu^{2}<1 / 4$, (b) $1 / 4<\nu^{2}<1 / 3$, and (c) $1 / 3<\nu^{2}$. By the horizontal lines, the diagram is divided into regions (d) $\alpha^{2}<4 / 27$, (e) $4 / 27<\alpha^{2}<8 / 27$, and (f) $8 / 27<\alpha^{2}$. The vertical (horizontal) arrows show the fixed values of the parameter $\nu(\alpha)$ taken from regions (a), (b), and (c) ((d), (e), and (f)) for which the $\left|A_{0}\right|$ versus $\alpha\left[\mid A_{0}\right.$ (versus $\nu$ ) characteristics are presented in Figs. 3(a), (b), and (c) ((d), (e), and (f)), respectively. The solid dot $(\nu, \alpha)=(0.9,0.6)$ shows the sets of parameters which will be used in the following analysis to demonstrate the delayed feedback control performance.

with respect to $s$. Knowing $s$, from Eq. (9) one can determine the steady state value of $z$,

$$
z_{0}=\frac{-\alpha}{s-1+\mathrm{i} \nu} .
$$

Solutions of the cubic equation (11) define stationary periodic orbits of the forced system. The period of these orbits coincide with the period $T$ of the external force, and the amplitude (the radius in the $(x, y)$ plane) is

$$
\left|A_{0}\right|=2\left|z_{0}\right|=2 \sqrt{s} .
$$

Equation (11) has three real roots provided

$$
\begin{aligned}
& \alpha_{1}^{2}(\nu)<\alpha^{2}<\alpha_{2}^{2}(\nu), \\
& \alpha_{1,2}^{2}(\nu)=\frac{2}{27}\left[9 \nu^{2}+1 \mp\left(1-3 \nu^{2}\right)^{3 / 2}\right],
\end{aligned}
$$

or one real root otherwise. Thus, the forced van der Pol oscillator has either three or one periodic orbit(s). The bifurcation diagram of Eq. (8) for $\kappa=0$ is shown in Fig. 2. Since it is symmetrical with respect to the $\nu$

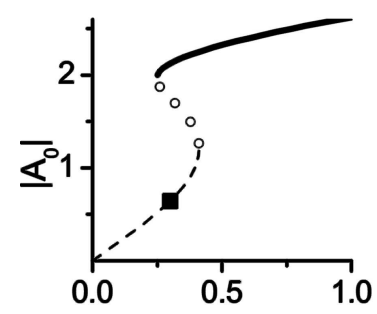

(a)
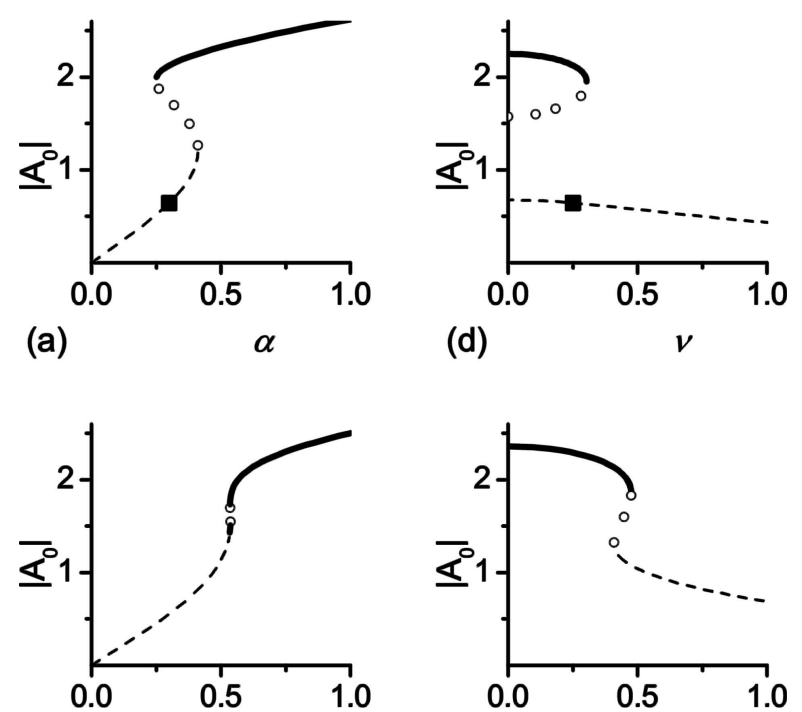

(b)

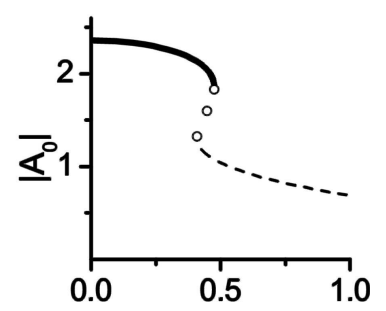

(e)

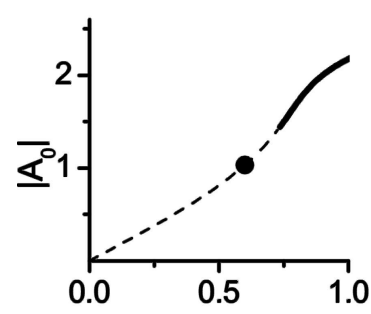

(c)

$\alpha$

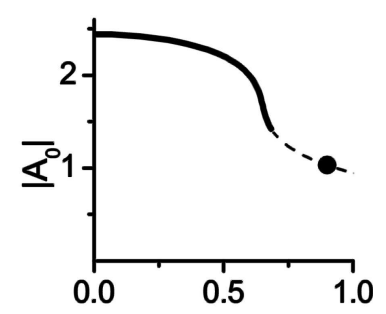

(f)

$\boldsymbol{V}$

Fig. 3. The amplitude $\left|A_{0}\right|$ of the periodic orbit as function of the amplitude $\alpha$ of the external force for the fixed value of the detuning (a) $\nu=0.25$, (b) $\nu=0.53$, (c) $\nu=0.9$, and as function of the detuning $\nu$ for the fixed value of the amplitude of the external force (d) $\alpha=0.3$, (e) $\alpha=0.46$, (f) $\alpha=0.6$. Solid lines denote the stable orbits, open circles represent the saddle orbits, and dashed lines show unstable periodic orbits with a pair of complex conjugate Floquet exponents. Solid dots mark the same set of the parameters $(\nu, \alpha)$ as in Fig. 2.

and $\alpha$ axes, only the part $\nu \geq 0, \alpha \geq 0$ is presented. The region with three orbits is between broken lines. Outside this region there is only one periodic orbit.

From a physical point of view it is interesting to investigate the bifurcations for two different cases, namely (i) for a fixed detuning $\nu$ and variable amplitude of external force $\alpha$, or (ii) for a fixed $\alpha$ and variable $\nu$. For the first case, the thin vertical lines divide the bifurcation diagram into three regions ((a), (b), and (c)) with different behaviour. The $\left|A_{0}\right|$ versus $\alpha$ characteristics in these three regions are shown in Figs. 3(a), (b), and (c) respectively. Similarly, for the second case the bifurcation diagram is divided into three regions ((d), (e), and (f)) by horizontal lines for which the $\left|A_{0}\right|$ versus $\nu$ characteristics are presented in Figs. 3(d), (e) and (f) respectively. Typical evolution of the periodic orbits in the $(x, y)$ plane is shown in Fig. 4. 
(a)
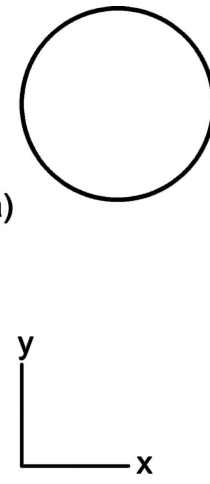

(b)

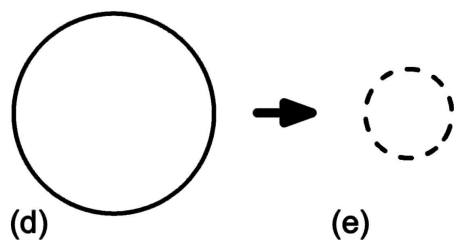

Fig. 4. Typical evolution of periodic orbits in the $(x, y)$ plane. Similarly as in Fig. 3 the solid lines, open circles, and dashed lines show the stable, saddle-type, and unstable (with a pair of complex conjugate FE's) orbits respectively. The scenario (a) $\rightarrow$ (b) $\rightarrow$ (c) is typical when passing the region with three solutions (see Figs. 3(a) and (e)). After two saddle-node bifurcations the stable orbit is replaced by an unstable one. The scenario (d) $\rightarrow$ (e) represents the Hopf bifurcation (see Figs. 3(c) and (f)).

To determine the stability of periodic orbits, we have to linearize Eq. (8), which leads to the characteristic equation

$$
\left(\frac{2 \lambda}{\varepsilon}\right)^{2}-2(1-2 s) \frac{2 \lambda}{\varepsilon}+f_{\nu}^{\prime}(s)=0 .
$$

Here $\lambda$ is the Floquet exponent (FE) of the periodic orbit, $s$ is the solution of the cubic equation (11), and

$$
f_{\nu}^{\prime}(s)=(3 s-1)(s-1)+\nu^{2}
$$

is the derivative of the function $f_{\nu}(s)$ defined in Eq. (10). The stability of a periodic orbit depends on the value of $s$ or, due to the relation (13) $\left|A_{0}\right|=2 \sqrt{s}$, on the amplitude of the orbit.

Two different types of bifurcations may occur in the system. For $f_{\nu}^{\prime}(s)=0$ we have a tangent (saddlenode) bifurcation, and for $s=1 / 2$ a Hopf bifurcation arises. The condition $f_{\nu}^{\prime}(s)=0$ defines the boundaries $\alpha^{2}=\alpha_{1,2}^{2}(\nu)$ of the region with three periodic orbits in the $(\nu, \alpha)$ plane (broken lines in Fig. 2). When crossing into this region two additional orbits of saddle and node types occur. The saddle orbit has two real FE's of different signs. The positive exponent $\lambda>0$ corresponds to the real positive Floquet multiplier $\mu=\mathrm{e}^{\lambda T}>1$ and thus this orbit satisfies the odd number property. Such an orbit cannot be stabilized by the usual delayed feedback method and we do not consider its control in this paper. The saddle orbits are marked by open circles in Figs. 3 and 4.

The condition of the Hopf bifurcation $s=1 / 2$ defines the minimal amplitude of the stable orbit $A_{\min }=$ $\sqrt{2}$. The orbits with amplitude $\left|A_{0}\right|<A_{\min }$ are un-

stable. In the $(\nu, \alpha)$ plane, this condition defines the hyperbola

$$
\alpha^{2}=f_{\nu}\left(\frac{1}{2}\right)=\frac{\nu^{2}}{2}+\frac{1}{8},
$$

which is shown by a solid line in Fig. 2. Above this line the oscillator is synchronized with the external force; the phase of the oscillator is locked by the phase of the external force and its amplitude is independent of time. Below this line, in the region of a single solution, the stability of the periodic orbit with the frequency $\omega$ is lost and we usually have a quasi-periodic behaviour. The orbits losing their stability through the Hopf bifurcation (scenario (d) $\rightarrow$ (e) in Fig. 4) have a pair of complex conjugate exponents with the positive real part. Similar properties has one of the orbits arising from the saddle-node bifurcation (scenario (a) $\rightarrow$ (b) $\rightarrow$ (c) in Fig. 4). Unstable orbits having a pair of complex conjugate exponents with the positive real part are marked by dashed lines in Figs. 3 and 4. In the next section we analyse their stability under action of the delayed feedback control.

\section{Linear stability of the system controlled by delayed feedback}

We now analyse Eq. 8 for $\kappa \neq 0$. The term $\kappa\left(z-z_{T}\right)$ does not change the steady state solutions of this equation, but can change their stability. Thus the delayed feedback can non-invasively influence the frequency entrainment condition. The characteristic equation now reads:

$$
\begin{aligned}
& \left(\frac{2 \lambda}{\varepsilon}\right)^{2}-2(1-2 s) \frac{2 \lambda}{\varepsilon}+(3 s-1)(s-1) \\
& +\left[\nu+\kappa\left(1-e^{-\lambda T}\right)\right]^{2}=0
\end{aligned}
$$

In a general case, this is a rather complex transcendental equation that has an infinite number of solutions. However, we can expect that close to the Hopf bifurcation the leading Floquet exponents will be proportional to the small parameter $\varepsilon$. This assumption allows the approximation $\mathrm{e}^{-\lambda T} \approx 1-\lambda T$, which simplifies Eq. 18:

$$
\left(1+K^{2}\right)\left(\frac{2 \lambda}{\varepsilon}\right)^{2}-2(1-2 s-\nu K) \frac{2 \lambda}{\varepsilon}+f_{\nu}^{\prime}(s)=0 .
$$

Here we use the notation

$$
K=\kappa T \frac{\varepsilon}{2}=k \frac{\pi}{\omega^{2}} .
$$




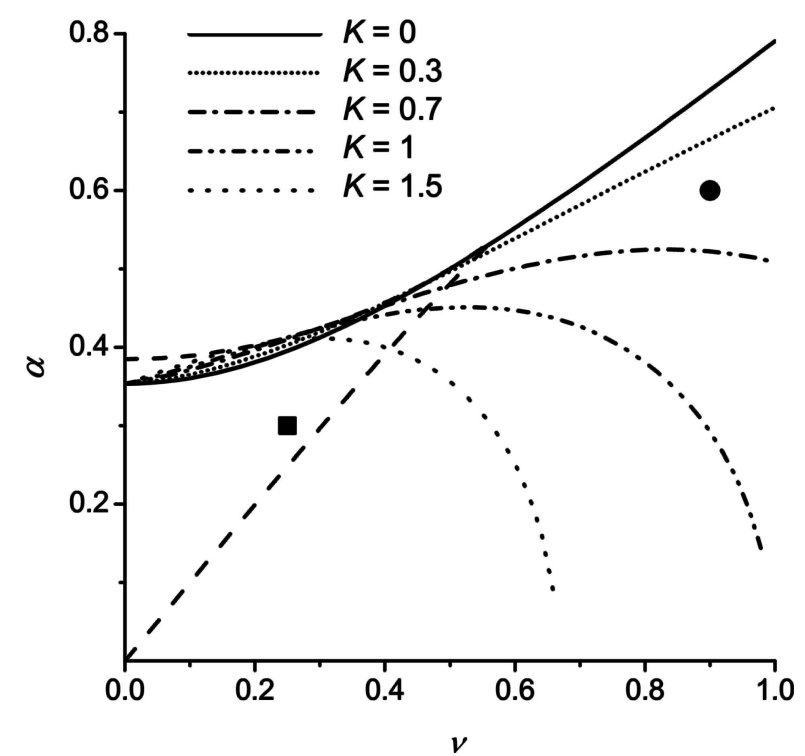

Fig. 5. The bifurcation diagram for van der Pol oscillator controlled by delayed feedback. The solid line defines the Hopf bifurcation for the uncontrolled system (the same as in Fig. 2), and broken lines are defined by Eq. (22). Above these lines the oscillator is synchronized with the external force.

This approximation is equivalent to that of replacing the delay term in Eq. (8) by the first derivative, $z_{T}=$ $z(t-T) \approx z(t)-T \dot{z}$. Such an approximation transforms the delay-differential equation (8) into the ordinary one

$$
\left(\frac{2}{\varepsilon}+\mathrm{i} \kappa T\right) \dot{z}=-\mathrm{i} \nu z-z\left(|z|^{2}-1\right)-\alpha .
$$

After linearization it yields Eq. (19).

From Eq. (19) we see that the delayed feedback changes the condition of the Hopf bifurcation, $1-2 s-$ $\nu K=0$, which now depends on the delayed feedback strength $K$. At the bifurcation point we have $s=(1-\nu K) / 2$. Substituting this in Eq. (11) and using Eq. (10) we obtain the relation between $K, \nu$, and $\alpha$ :

$$
\alpha^{2}=\frac{1}{8}(1-\nu K)\left[(1+\nu K)^{2}+4 \nu^{2}\right] .
$$

In Fig. 5, these relations are presented by curves in the $(\nu, \alpha)$ plane for different fixed values of $K$. These curves define the boundaries of synchronization for the controlled oscillator. Above these curves the oscillator is synchronized with the periodic force. We see that the delayed feedback perturbation extends the phase locked domain in the Arnold tongue.

For a fixed value of the parameters $(\nu, \alpha)$, the threshold of the feedback strength at which the Hopf bifurcation occurs is $K_{0}=(1-2 s) / \nu$, where $s$ satisfies the cubic equation (11). Employing Eq. (20) and relation $s=\left|A_{0}\right|^{2} / 4$ the threshold can be presented in the form

$$
k_{0}=\frac{\omega^{2}}{\pi} K_{0}=\frac{\omega^{2}}{\pi \nu}\left(1-\frac{\left|A_{0}\right|^{2}}{2}\right) .
$$

To demonstrate how the Floquet exponents depend on the control gain $k$ we specify the parameters $(\nu, \alpha)$ to be $(0.9,0.6)$. This set of parameters is marked by solid dots in Figs. 2, 3, and 5. We have calculated the leading Floquet exponents of the initially unstable orbit using three different methods, namely, (i) solving transcendental equation (18), (ii) using quadratic equation (19), and (iii) solving exact (nonaveraged) linearized system (2). Equation (18) have been solved by the Newton-Raphson algorithm. The third method is based on the numerical analysis of the variational equations

$$
\begin{aligned}
\delta \dot{x}= & \delta y \\
\delta \dot{y}= & -\left(\omega_{0}^{2}-2 \varepsilon x_{0} y_{0}\right) \delta x-\varepsilon\left(x_{0}^{2}-1\right) \delta y \\
& +k\left(\delta x-\delta x_{T}\right)
\end{aligned}
$$

derived from the system (2). Here $\delta x=x-x_{0}(t)$, $\delta y=y-y_{0}(t)$ are small deviations from the unstable periodic orbit $\left[x_{0}(t), y_{0}(t)\right]=\left[x_{0}(t+T), y_{0}(t+T)\right]$ that satisfies the uncontrolled system (2). The leading Lyapunov exponents of this system have been calculated according to the algorithm described in Ref. [5]. Note that the Lyapunov exponent of a periodic orbit coincides with the real part of the Floquet exponent.

The results of the above analysis for two different values of the parameter $\varepsilon$ equal to 0.01 and 0.1 are presented in Fig. 6. The exact values of the leading FE's determined from Eq. (24) are shown by dots. There are two branches (the left-hand and the right-hand) defining the interval of stability $k_{0}<k<k_{1}$ in which the real part of the leading FE is negative. The parameters $k_{0}$ and $k_{1}$ denote the lower and upper control thresholds, respectively.

First we discuss the results for the left-hand branch. For $\varepsilon=0.01$, all the three above methods give quantitatively coinciding results (Fig. 6(a)). Thus for small $\varepsilon$ the leading FE of the left-hand branch can be reliably obtained from the simple quadratic equation (19), which yields

$$
\Re \lambda=\frac{\varepsilon}{2} \frac{1-\frac{\left|A_{0}\right|^{2}}{2}-\nu k \frac{\pi}{\omega^{2}}}{1+\left(k \frac{\pi}{\omega^{2}}\right)^{2}},
$$



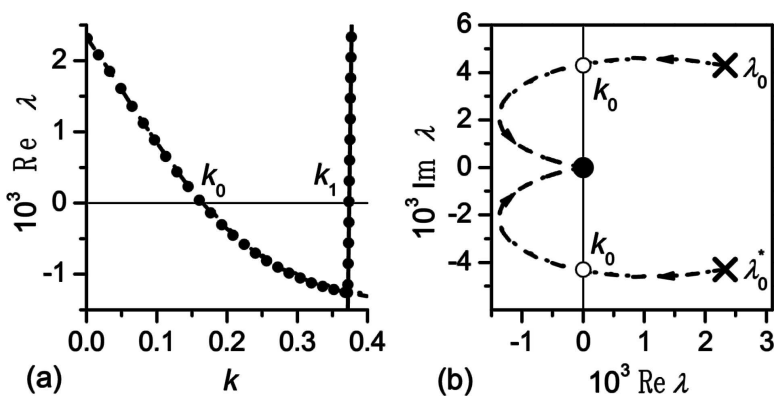

Fig. 6. Leading Floquet exponents as functions of the control gain for $(\nu, \alpha)=(0.9,0.6)$. Here and in all numerical demonstrations below we take $\omega_{0}=1$. By this it is meant that $\omega, \varepsilon$, and $\lambda$ are measured in units of $\omega_{0}$, time $t$ is measured in units of $\omega_{0}^{-1}$, and $k$ is measured in units of $\omega_{0}^{2}$. (a) $\Re \lambda$ versus $k$ for $\varepsilon=0.01$. For the given values of parameters $\nu, \alpha$, and $\varepsilon$, we have $\omega \approx 1.00451$ and $a \approx 0.01205$. The amplitude of the unstable orbit is $\left|A_{0}\right| \approx 1.034$ and its FE's for $k=0$ are $\lambda_{0} \approx(2.327 \pm 4.297 \mathrm{i}) \cdot 10^{-3}$. Solid dots are the values of the Lyapunov exponents obtained from exact variational equations (24). The dashed and dotted lines calculated from Eq. (18) and Eq. (19) (or Eq. (25) respectively approximate the left-hand branch. The solid line calculated from Eq. (28) approximates the right-hand branch. (b) Root loci of Eq. (18) (dotted line) and Eq. (19) (dashed line) as $k$ varies from 0 to $\infty$ for the same parameter $\varepsilon$ value as in (a). Crosses and black dot denote the location of the roots for $k=0$ and $k=\infty$, respectively.

and the threshold $k_{0}$ of the Hopf bifurcation is well described by Eq. (23). The transcendental equation (18) gives good results even for $\varepsilon=0.1$, while Eqs. (19) or (25) are less appropriate (Fig. 6(b)).

The right-hand branch of the FE defining the upper threshold $k_{1}$ cannot be quantitatively well described by Eq. (18). This is because the term $k\left(x-x_{T}\right)$ responsible for the control in the system (2) is not small in this case, and the averaging procedure performed with this system is not valid. Nevertheless, we can find an approximate analytical expression for the FE using exact (nonaveraged) variational equations (24). For the righthand branch, the nonlinear terms in Eq. (18) are small in comparison with the control term. Thus in the variational equations we can neglect the terms containing $\varepsilon$. Setting $\varepsilon=0$ in Eq. (24) we obtain the characteristic equation

$$
\lambda^{2}+\omega_{0}^{2}-k\left(1-\mathrm{e}^{-\lambda T}\right)=0 .
$$

Root loci diagram of the relevant branch for this equation when varying $k$ is shown in Fig. 7. The pair of complex conjugate roots intersects the imaginary axes at the points $\lambda= \pm \mathrm{i} \pi / T= \pm \mathrm{i} \omega / 2$. This intersection appears for $k=k_{1}$, where

$$
k_{1}=\frac{1}{2}\left(\omega_{0}^{2}-\frac{\omega^{2}}{4}\right)
$$

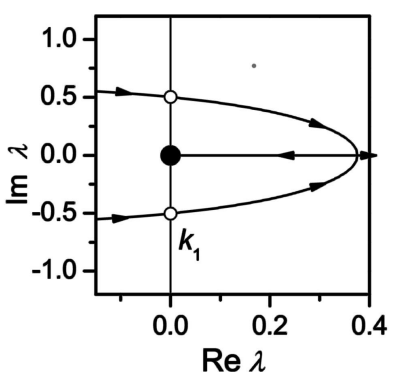

Fig. 7. Root loci of Eq. (26) for $\omega=1.00451\left(\omega_{0}=1\right)$.

defines the upper threshold of stability. For $k=k_{1}$, the orbit loses stability by a period doubling bifurcation since the intersection of the imaginary axes appears at a half frequency of the external force, $\lambda= \pm i \omega / 2$. Expanding the solution of Eq. (26) in Taylor series close to the threshold $k=k_{1}$, we obtain an approximate analytical expression

$$
\operatorname{Re} \lambda=\frac{4 \pi \frac{k_{1}}{\omega}}{\omega^{2}+\left(2 \pi \frac{k_{1}}{\omega}\right)^{2}}\left(k-k_{1}\right)
$$

that describes well the $\operatorname{Re} \lambda$ versus $k$ dependence for the right-hand branch (Fig. 6(a,b)).

Having analytical expressions for the left-hand (Eq. (25)) and right-hand (Eq. (28)) branches one can easily evaluate an optimal value $k_{\mathrm{op}}$ of the control gain that provides the minimal $\Re \lambda$ and thus the fastest convergence to the desired orbit. This value is defined by a simple intersection of these two branches and can be found from a cubic equation with respect to $k$, which results from equating Eq. (25) and Eq. (28).

\section{Numerical demonstrations}

To verify the validity of the linear theory we have numerically investigated the original nonlinear differential equations (2). For the set of parameters $(\nu, \alpha)=$ $(0.6,0.9), \varepsilon=0.1$ the results are presented in Fig. 8. Without control $(t<80 T)$ the van der Pol oscillator is not synchronized with the external force and a beat phenomenon is observed (Fig. 8(a)). The DFC perturbation is switched on at the moment $t_{c}=80 T$; it stabilizes an unstable UPO and we have a periodic motion synchronized with an external force (Fig. 8(b)). Whenever the synchronization is established the feedback perturbation vanishes (Fig. 8(c)). The envelopes of the transient are well described by the averaged amplitude equation (8). This confirms the validity of the averaging procedure applied to the time-delay system (2). 

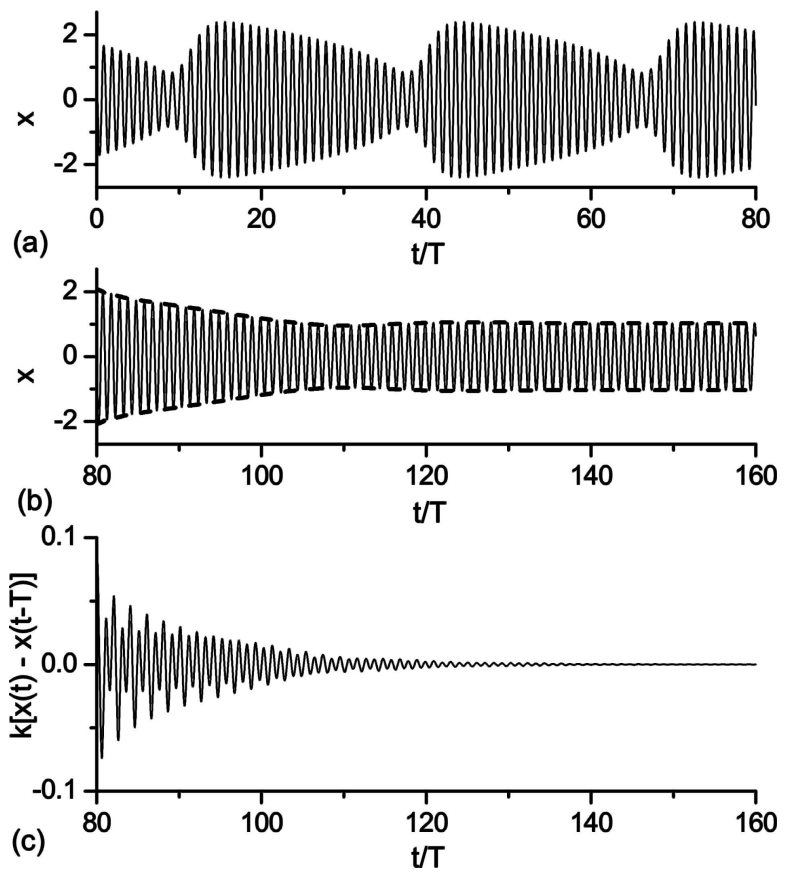

Fig. 8. Results of numerical integration of delay-differential equations (2) for $(\nu, \alpha)=(0.9,0.6), \varepsilon=0.1$. (a) Dynamics of the $x$ variable without control. (b,c) Dynamics of the $x$ variable and perturbation $k[x(t)-x(t-T)]$ when the control is switched on. The broken line (an envelope) in (b) is the dynamics of the complex amplitude $|A(t)|=2|z(t)|$ obtained from averaged equation (8). The strength of the feedback gain is $k=0.34$, other parameters are the same as in Fig. 6(c).

\section{Conclusions}

We have developed an analytical approach for the delayed feedback control of a forced self-sustained oscillator close to a supercritical Hopf bifurcation. The analytical approach is based on an averaging method, a classical asymptotic method of nonlinear dynamics developed for weakly nonlinear oscillators. We have shown that this method works well even in the presence of the delayed feedback.

Our analysis shows that the domain of synchronization of a forced self-sustained oscillator can be essentially extended by delayed feedback. This extension is based on the stabilization of the existing unstable periodic orbit and is attained with tiny control perturbations.

In this paper, the delayed feedback control method is applied for the first time to control a quasi-periodic motion, i. e. the motion on a torus in the phase space. The unstable periodic orbits in this case have a pair of complex conjugate Floquet multipliers outside the unit circle in the complex plane.

The analytical approach is demonstrated for the paradigmatic system of the forced van der Pol oscil- lator. We have obtained simple analytical expressions for the dependence of leading Floquet exponents on the control gain and determined the lower and upper threshold of stability as well as an optimal value of the control gain. The main results and the approach are of general importance since they are relevant to any forced self-sustained oscillator close to the supercritical Hopf bifurcation. We believe that the developed analytical approach is an important contribution to the theory of the delayed feedback control.

\section{References}

[1] K. Pyragas, Continuous control of chaos by selfcontrolling feedback, Phys. Lett. A 170, 421-428 (1992).

[2] Handbook of Chaos Control, ed. H.G. Shuster (WileyVCH, Weinheim, 1999).

[3] W. Just, T. Bernard, M. Ostheimer, E. Reibold, and H. Benner, Mechanism of time-delayed feedback control, Phys. Rev. Lett. 78, 203-206 (1997).

[4] K. Pyragas, Analytical properties and optimization of time-delayed feedback control, Phys. Rev. E 66, 026207-1-9 (2002).

[5] K. Pyragas, Control of chaos via extended delay feedback, Phys. Lett. A 206, 323-330 (1995). 


\section{PRIVERSTINIŲ SAVAIMINIU VIRPESIU SINCHRONIZACIJOS VALDYMAS}

T. Pyragienè, K. Pyragas

Puslaidininkiu fizikos institutas, Vilnius, Lietuva

\section{Santrauka}

Vèluojantis grįžtamasis ryšys taikomas kvaziperiodiniam judejjimui valdyti. Nagrinejjame silpnai netiesini van der Polio osciliatorių, žadinamą išorine periodine jèga. Sistema, esanti arti superkritinės Hopfo bifurkacijos, ištirta analiziškai, pasitelkus vidurki- nimo metodą. Parodyta, kad vèluojantis grižtamasis ryšys žymiai praplečia osciliatoriaus sinchronizacijos ribas. Nors metodas pademonstruotas pasirinktai fizikinei sistemai - van der Polio osciliatoriui, jis tinka bet kuriai savaiminių virpesių sistemai, kai ji yra arti superkritinès Hopfo bifurkacijos. 